\title{
Approximation Hardness of the Steiner Tree Problem on Graphs
}

\author{
Miroslav Chlebík ${ }^{1}$ and Janka Chlebíková ${ }^{2 \star}$ \\ 1 Max Planck Institute for Mathematics in the Sciences \\ Inselstraße 22-26, D-04103 Leipzig, Germany \\ ${ }^{2}$ Christian-Albrechts-Universität zu Kiel \\ Institut für Informatik und Praktische Mathematik \\ Olshausenstraße 40, D-24098 Kiel, Germany \\ jch@informatik.uni-kiel.de
}

\begin{abstract}
Steiner tree problem in weighted graphs seeks a minimum weight subtree containing a given subset of the vertices (terminals). We show that it is NP-hard to approximate the Steiner tree problem within 96/95. Our inapproximability results are stated in parametric way and can be further improved just providing gadgets and/or expanders with better parameters. The reduction is from Håstad's inapproximability result for maximum satisfiability of linear equations modulo 2 with three unknowns per equation. This was first used for the Steiner tree problem by Thimm whose approach was the main starting point for our results.
\end{abstract}

\section{Introduction}

Given a graph $G=(V, E)$, a weight function $w: E \rightarrow \mathbb{R}^{+}$on the edges, and a set of required vertices $T \subseteq V$, the terminals. A Steiner tree is a subtree of $G$ that spans all vertices in $T$. (It might use vertices in $V \backslash T$ as well.)

The Steiner Tree Problem (STP) is to find a Steiner tree of minimum weight. Denote OPT the optimal value of the Steiner tree OPT $:=\min \{w(\mathcal{T})$ : $\mathcal{T}$ is a Steiner tree\}.

An instance of the Steiner tree problem is called quasi-bipartite if there is no edge in the set $V \backslash T$, and uniformly quasi-bipartite if it is quasi-bipartite and edges incident to the same non-terminal vertex have the same weight.

Steiner trees are important in various applications, for example VLSI routing, wirelength estimation and network routing.

The Steiner Tree Problem is among the 21 basic problems for which Karp has shown NP-hardness in his paper [6].

As we cannot expect to find polynomial time algorithms for solving it exactly (unless $\mathrm{P}=\mathrm{NP}$ ), the search is for effective approximation algorithms. During the last years many approximation algorithms for the STEIner Tree Problem were designed, see [5] for survey. The currently best approximation algorithm

\footnotetext{
* The second author has been supported by the EU-Project ARACNE, Approximation
} and Randomized Algorithms in Communication Networks, HPRN-CT-199-00112. 
of Robins and Zelikovsky ([8]) has a performation ratio of 1.550, and 1.279 for quasi-bipartite instances. In the case of uniformly quasi-bipartite instances, the best known algorithm has a performation ratio 1.217 [5].

It is a natural question how small the performation ratio of a polynomial time algorithm can get. Unless $\mathrm{P}=\mathrm{NP}$, it cannot get arbitrarily close to 1 . This follows from PCP-Theorem [1] and from the fact that the problem is APXcomplete [2].

Until now, the best known lower bound is inapproximability within 1.00617, due to Thimm ([9]). (In fact, Thimm's paper claims the lower bound of 1.0074, but there is a small error in the paper and only a slightly worse lower bound can be shown along the lines of the proof. Moreover, Thimm's paper uses the more restrictive assumption co-RP $\neq \mathrm{NP}$.)

\section{Main Result}

The main result of this article improves the lower bounds on approximability of the STP and reduces the gap between known approximability and inapproximability results.

Main Theorem. It is NP-hard to approximate the Steiner Tree Problem within ratio $1.01063\left(>\frac{96}{95}\right)$. For the special case of (uniformly) quasi-bipartite instances approximation within ratio $1.00791\left(>\frac{128}{127}\right)$ is NP-hard.

Our reduction is from Håstad's hard-gap result for maximum satisfiability of linear equations modulo 2 with three unknowns per equation, MAX-E3-Lin-2.

Definition 1. MAX-E3-Lin-2 is the following optimization problem: Given a system of linear equations over $\mathbb{Z}_{2}$, with exactly 3 variables in each equation. The goal is to find an assignment to the variables that satisfies as many equations as possible.

To suit our purposes we state Håstad's important result in the following way (see also [7] for application of that result in a similar context).

Theorem 1. ([4]) For every $\varepsilon \in\left(0, \frac{1}{4}\right)$ and every fixed sufficiently large integer $k \geq k(\varepsilon)$, the following partial decision subproblem of MAX-E3-Lin-2 is NP-hard:

$P(\varepsilon, k)\left\{\begin{array}{l}\text { Given an instance MAX-E3-LIN- } 2 \text { consisting of } n \text { equations and } \\ \text { with exactly } 2 k \text { occurrences of each variable, to decide if at least } \\ (1-\varepsilon) n \text { or at most }\left(\frac{1}{2}+\varepsilon\right) n \text { equations are satisfied by the optimal } \\ \text { assignment. }\end{array}\right.$

The same NP-hardness result holds on instances where all equations are of the form $x+y+z=0$ (respectively, all equations are of the form $x+y+z=1$ ), where literals $x, y, z$ are variables or their negations, and each variable appears exactly $k$ times negated and $k$ times unnegated. This subproblem of the problem $P(\varepsilon, k)$ will be referred to as $P_{0}(\varepsilon, k)$ (respectively $P_{1}(\varepsilon, k)$ ) in what follows. 


\section{NP-hard-gap Preserving Reduction}

We start with a set $L$ of $n$ linear equations over $\mathbb{Z}_{2}$, all of the form $x+y+z=0$ (respectively, all of the form $x+y+z=1$ ), where literals $x, y, z$ are variables from the set $\mathcal{V}$ or their negations, and each variable $v \in \mathcal{V}$ appears in $L$ exactly $k$ times negated as $\bar{v}$ and $k$ times unnegated.

For an assignment $\psi \in\{0,1\}^{\mathcal{V}}$ to variables let $S(\psi)$ be the number of equations of $L$ satisfied by $\psi$. We will reduce the problem of maximizing $S(\psi)$ over all assignments to the instance of the Steiner Tree Problem. To get an approximation preserving reduction we will use equation gadgets and couple them properly using a graph with good vertex-expansion property.

\section{The Equation Gadget}

Now we introduce the notion of $(\alpha, \beta, \gamma)$-gadget for the reduction from the equation system of the form $x+y+z=0$ (respectively, from the system of the form $x+y+z=1)$. This will be an instance $G=(V, E), w: E \rightarrow \mathbb{R}^{+}, T \subseteq V$ of the Steiner Tree Problem with the following properties:

1. One of (possibly more) terminal vertices is distinguished and denoted by $\mathrm{O}$.

2. Three of (possibly more) non-terminal vertices are distinguished and denoted by $x, y$ and $z$.

3. For any $u \in\{x, y, z\}$ there is a path from $u$ to $\mathrm{O}$ of weight at most 1 .

4. For any subset $R$ of $\{x, y, z\}$ consider the instance of the STP with altered terminal set $T_{R}:=T \cup R$. The weight of the corresponding minimum Steiner tree is denoted by $s_{R}$ and is required to depend on the cardinality of the set $R$ only in the following way,

$$
s_{R}=\alpha+|R| \beta+(|R| \bmod 2) \gamma .
$$

(Respectively, if our system $L$ is of the form $x+y+z=1$, we require $\left.s_{R}=\alpha+|R| \beta+(1-|R| \bmod 2) \gamma.\right)$

An $(\alpha, \beta, \gamma)$-gadget with no edges between non-terminal vertices is called quasi-bipartite $(\alpha, \beta, \gamma)$-gadget. A quasi-bipartite $(\alpha, \beta, \gamma)$-gadget such that edges incident to the same non-terminal have the same weight and for vertices $x, y, z$ the incident edges have the weight 1 is called uniformly quasi-bipartite $(\alpha, \beta, \gamma)$ gadget.

In our reduction we will use one copy of a fixed $(\alpha, \beta, \gamma)$-gadget per each equation of $L$. For each variable $v, k$ negated and $k$ unnegated occurrences of $v$ will be further coupled using a particular $k$ by $k$ regular bipartite multigraph, which is a good expander.

The condition 3 above is just a proper normalization.

The condition 4 on $s_{k}:=s_{R}, k=|R| \in\{0,1,2,3\}$, has the following interpretation in our construction: $\alpha$ is a basic cost per equation, $\beta$ is an extra payment for connecting some of $\{x, y, z\}$ to the Steiner tree, and $\gamma$ is a penalty for the failure in the parity check of the number of vertices of $\{x, y, z\}$ adjacent to the Steiner tree. 
Example 1. For any $\gamma \in\left(0, \frac{1}{4}\right\rangle$ there is a $(0,1-\gamma, \gamma)$-gadget (for the system $L$ of the form $x+y+z=0$ ), depicted on Fig. 1. The vertex $\mathrm{O}$ is the only terminal. Clearly $s_{0}=0, s_{1}=1, s_{2}=2-2 \gamma$, and $s_{3}=3-2 \gamma$.

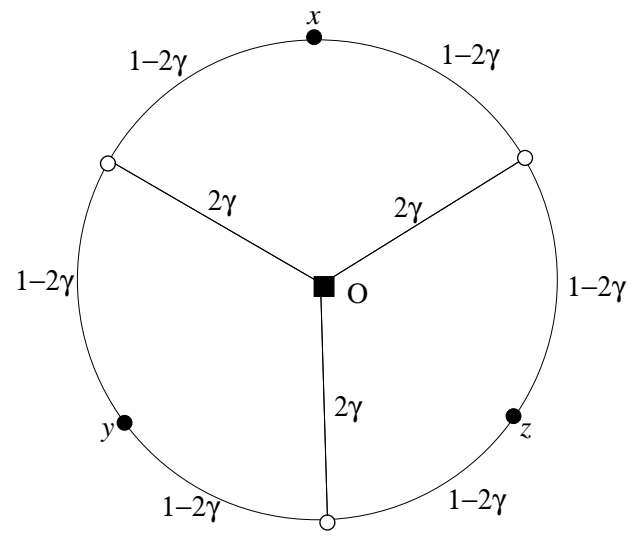

Fig. 1.

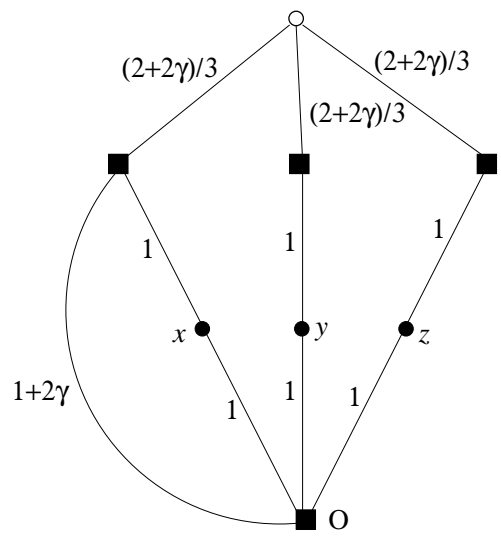

Fig. 2.

Example 2. For any $\gamma \in\left\langle 0, \frac{1}{2}\right\rangle$ there is a uniform quasi-bipartite $(3+3 \gamma, 1-\gamma, \gamma)$ gadget (for the system $L$ of the form $x+y+z=1$ ), depicted on Fig. 2 .

There are 4 terminals in this gadget, all drawn as boxes. One can easily check that $s_{0}=3+4 \gamma, s_{1}=4+2 \gamma, s_{2}=5+2 \gamma$, and $s_{3}=6$. This is essentially the gadget used by Thimm ([9]) in his reduction translated to our language.

\section{Expanders}

An expander with parameters $(c, \tau, d)$ (shortly, $(c, \tau, d)$-expander) is a $d$-regular bipartite multigraph with balanced $k$ by $k$ bipartition $\left(V_{1}, V_{2}\right)$, such that

$$
\text { if } U \subseteq V_{1} \text { or } U \subseteq V_{2} \text {, and }|U| \leq \tau k \text {, then }|\Gamma(U)| \geq c|U| \text {. }
$$

Here $\Gamma(U)$ stands for the set of neighbors of $U, \Gamma(U):=\{y: y$ is a vertex adjacent to some $x \in U\}$.

It is known that for any sufficiently large $k,(c, \tau, d)$-expander with $k$ by $k$ bipartition exists, provided that $0<\tau<\frac{1}{c}<1$ and $H_{d}(c, \tau)>0$, where

$$
H_{d}(c, \tau):=(d-1) F(\tau)-d c \tau F\left(\frac{1}{c}\right)-F(c \tau),
$$

with $F(x)=-x \log x-(1-x) \log (1-x)$ being the entropy function. In fact, under the above condition, almost every random $d$-regular balanced bipartite multigraph is $(c, \tau, d)$-expander, see Theorem 6.6 in [3]. 
Definition 2. We say that d-regular bipartite graph with $k$ by $k$ bipartition $\left(V_{1}, V_{2}\right)$ is a c-good expander provided the following implication holds:

$$
\text { if } U \subseteq V_{1} \text { or } U \subseteq V_{2} \text {, then }|\Gamma(U)| \geq \min \{c|U|, k+1-|U|\} .
$$

The condition of being $c$-good expander for a balanced $d$-regular bipartite graph is just a bit stronger than the one of being $\left(c, \frac{1}{c+1}, d\right)$-expander. In particular, it can be easily seen that $(c, \tau, d)$-expander with $k$ by $k$ bipartition is $c$-good, provided that $\tau>\frac{1}{c+1}$ and $k \geq \frac{c}{(c+1) \tau-1}$.

Consequently, for any sufficiently large $k$, a $d$-regular $c$-good expander with $k$ by $k$ bipartition exists, provided that $c>1$ satisfies

$$
H_{d}\left(c, \frac{1}{c+1}\right)>0 .
$$

In fact, by continuity it follows that $H_{d}(c, \tau)>0$ also for some $\tau \in\left(\frac{1}{c+1}, \frac{1}{c}\right)$, and we can use the existence result for $(c, \tau, d)$-expanders cited above.

For any integer $d \geq 3$ we introduce the constant $c(d)$ defined in the following way:

$$
c(d)=\sup \{c: \text { there are infinitely many } d \text {-regular } c \text {-good expanders }\} .
$$

Denote by $x(d)$ the unique $x \in(1, \infty)$ for which $H_{d}\left(x, \frac{1}{x+1}\right)=0$. It can be easily numerically approximated, as $(x+1) H_{d}\left(x, \frac{1}{x+1}\right)$ can be simplified to

$$
(d-2)(x+1) \log (x+1)-(2 d-2) x \log x+d(x-1) \log (x-1) .
$$

Hence (1) holds for any $c$ in $(1, x(d))$ and, consequently, $c(d) \geq x(d)$ for any integer $d \geq 3$. In particular, $c(6)>1.76222$ and $c(7)>1.94606$.

Now we are ready to describe our reduction of instances like $L$ to the instances of the Steiner Tree Problem. For this purpose we will use one fixed $(\alpha, \beta, \gamma)$ gadget, and one fixed $k$ by $k$ bipartite $d$-regular multigraph $H$ which is supposed to be $\frac{\beta+\gamma}{\beta-\gamma}$-good.

\section{Construction}

Take $n$ pairwise disjoint copies of that $(\alpha, \beta, \gamma)$-gadget, one for each equation of the system $L$, and identify their vertices labeled by $\mathrm{O}$. The $x, y, z$ vertices in each equation gadget correspond to occurrences of literals in that equation and we re-label them by those literals. By assumption, each variable from $\mathcal{V}$ appears exactly $k$ times negated and $k$ times unnegated as a label. We couple negated and unnegated occurrences of each variable using our fixed bipartite $d$-regular multigraph $H$ with bipartition $\left(V_{1}, V_{2}\right), V_{1}=\left\{a_{1}, a_{2}, \ldots, a_{k}\right\}, V_{2}=$ $\left\{b_{1}, b_{2}, \ldots, b_{k}\right\}$ in the following way:

Assume that equations (and their equation gadgets) are numbered by $1,2, \ldots$, $n$. Given literal $x$, i.e. $x=v$ or $x=\bar{v}$ for some $v \in \mathcal{V}$, let $m_{1}(x)<m_{2}(x)<$ $\cdots<m_{k}(x)$ be the numbers of equations in which that literal occurs. 
Consider one variable of $\mathcal{V}$, say $v$. For each $a_{i} b_{j}$ edge $e$ of $H(1 \leq i, j \leq k)$ we add a new coupling terminal vertex $t(v, e)$. Now connect it with the $v$-vertex in the $m_{i}(v)$-th equation gadget and with the $\bar{v}$-vertex in the $m_{j}(\bar{v})$-th equation gadget, by edges of weight 1 .

Making the above coupling for all variables from $\mathcal{V}$ one after another, we get an instance of the Steiner tree problem, that corresponds to the system $L$. Consider any Steiner tree $\mathcal{T}$ for this instance, i.e. a tree spanning all terminals.

In the following claim we prove that in the Steiner trees with the optimal value OPT each coupling terminal vertex $t(v, e)$ is a leaf of $\mathcal{T}$. We call simple a Steiner tree $\mathcal{T}$ with mentioned property that each coupling terminal vertex $t(v, e)$ is a leaf of $\mathcal{T}$.

Claim. OPT $=\min \{w(\mathcal{T}): \mathcal{T}$ is a simple Steiner tree $\}$.

Proof. To show that, one can transform any given Steiner tree $\mathcal{T}$ with nonempty 'bad' set $\operatorname{BAD}(\mathcal{T}):=\{$ coupling terminals that are not leaves of $\mathcal{T}\}$ to another Steiner tree $\mathcal{T}^{\prime}$ with $\left|\operatorname{BAD}\left(\mathcal{T}^{\prime}\right)\right|<|\operatorname{BAD}(\mathcal{T})|$ and $w\left(\mathcal{T}^{\prime}\right) \leq w(\mathcal{T})$. Fix $\mathcal{T}$ with nonempty bad set and choose $t=t(v, e) \in \operatorname{BAD}(\mathcal{T})$. Deleting one of edges incident to $t$ decreases both $|\operatorname{BAD}(\mathcal{T})|$ and $w(\mathcal{T})$ by 1 . But we have two components now, with one of vertices labeled by $v$ or $\bar{v}$ in the distinct component than the vertex $\mathrm{O}$ belongs. Connect this vertex with $\mathrm{O}$ in its equation gadget in the cheapest possible way, to obtain the Steiner tree $\mathcal{T}^{\prime}$.

By property 3 of $(\alpha, \beta, \gamma)$-gadget it increases the weight by at most 1 , hence $w\left(\mathcal{T}^{\prime}\right) \leq w(\mathcal{T})$.

Definition 3. We say that a simple Steiner tree $\mathcal{T}$ is well-behaved if it is locally minimal in the following sense:

Consider any equation of $L$, say $i$-th, $i \in\{1,2, \ldots, n\}$. Let $x, y, z$ be its literals, $T:=T^{i}$ be the set of terminal vertices of its equation gadget, and $R:=R^{i}$ be the set of vertices of this gadget labeled by $x, y$, or $z$, that belong to $\mathcal{T}$. The subgraph $\mathcal{T}^{i}$ of $\mathcal{T}$ induced by this equation gadget is supposed to be the local minimal Steiner tree (in this gadget) for the altered terminal set $T_{R}:=T \cup R$.

Claim. OPT $=\min \{w(\mathcal{T}): \mathcal{T}$ is a well-behaved Steiner tree $\}$.

Proof. Clearly, any simple Steiner tree $\mathcal{T}$ with $w(\mathcal{T})=$ OPT has to be wellbehaved, because otherwise one could create, by local change in some of its gadget, a Steiner tree with less weight. In particular, OPT $=\min \{w(\mathcal{T}): \mathcal{T}$ is a well-behaved Steiner tree\}.

By property 4 of $(\alpha, \beta, \gamma)$-gadget, the weight of subtree $\mathcal{T}^{i}$ is $\alpha+|R| \beta+$ $(|R| \bmod 2) \gamma($ respectively, $\alpha+|R| \beta+(1-|R| \bmod 2) \gamma)$. Hence, the weight of any well-behaved Steiner tree $\mathcal{T}$ can be expressed in the following way: denote by $N$ the number of vertices corresponding to literals that belong to $\mathcal{T}$, and by $M$ the number of equations for which $R:=R^{i}$ above fails the parity check, i.e. $\left|R^{i}\right|$ is odd (respectively, $\left|R^{i}\right|$ is even). Then

$$
w(\mathcal{T})=\alpha n+\frac{3}{2} n d+N \beta+M \gamma
$$


Here $\frac{3}{2} n d$ edges of weight 1 connect all $\frac{3}{2} n d$ coupling terminals as leaves of the tree $\mathcal{T}$. Clearly, $N \geq \frac{3}{2} n$, as at least one from each coupled pair of vertices correspond to variables has to belong to $\mathcal{T}$, to connect the corresponding coupling terminal to the tree $\mathcal{T}$.

Suppose we are given an assignment $\psi \in\{0,1\}^{\mathcal{V}}$ to variables and let $S(\psi)$ be the number of equations satisfied by $\psi$. For $i$-th equation of $L(i=1,2, \ldots, n)$ let $R:=R_{\psi}^{i}$ denote the set of vertices in its equation gadget labeled by literals with value 1 by the assignment $\psi$, and let $T:=T^{i}$ denote the terminals of this equation gadget. Take one (of possibly more) local minimum Steiner tree in this gadget with altered terminal set $T_{R}:=T \cup R$ and connect each vertex of $R$ to all $d$ coupling terminals adjacent to it. Such kind of well-behaved Steiner tree (denoted by $\mathcal{T}_{\psi}$ ), which follows from some assignment $\psi$ will be called standard Steiner tree.

The weight of standard Steiner tree $\mathcal{T}_{\psi}$ can be expressed using (3), where we have now $N=\frac{3}{2} n$ (exactly half of vertices for variables correspond to literals assigned 1), and $M=n-S(\psi)$. Hence

$$
w\left(\mathcal{T}_{\psi}\right)=\alpha n+\frac{3}{2} n d+\frac{3}{2} n \beta+(n-S(\psi)) \gamma .
$$

The challenge is to prove Lemma 1 below that OPT is achieved on a standard Steiner tree for some assignment $\psi$. If this is the case, from (4) it can be easily seen that hard-gap result of Håstad for the problem max $S(\psi)$ implies the corresponding hard-gap and inapproximability results for the STEINER TreE Problem.

Lemma 1. If $(\alpha, \beta, \gamma)$-gadget has parameters $\beta>\gamma \geq 0$, and an expander graph used for the coupling is $\frac{\beta+\gamma}{\beta-\gamma}$-good, then

$$
O P T=\min \{w(\mathcal{T}): \mathcal{T} \text { is a standard Steiner tree }\} .
$$

Proof. We already know that there exists a well-behaved Steiner tree $\mathcal{T}$ such that $w(\mathcal{T})=$ OPT. Thus it is sufficient to show that $\mathcal{T}$ can be transformed into a standard Steiner tree $\mathcal{T}^{*}$ without increasing the weight. In the following we describe such construction of $\mathcal{T}^{*}$ from $\mathcal{T}$ in $|\mathcal{V}|$ steps. Consider one variable, $v \in \mathcal{V}$. Let $A_{1}$ be the set of vertices labeled by $v$, and $A_{2}$ be the set of vertices labeled by $\bar{v}$. Clearly $\left|A_{1}\right|=\left|A_{2}\right|=k$. Denote by $C_{i}(i=1,2)$ the set of vertices in $A_{i}$ that are vertices of the tree $\mathcal{T}$, and put $U_{i}=A_{i} \backslash C_{i}$. We will assume that $\left|U_{1}\right| \leq\left|U_{2}\right|$, otherwise we change the role of $A_{1}$ and $A_{2}$ in what follows.

Let $\Gamma(U)$, for a set $U \subseteq A_{1}$, be the set of vertices in $A_{2}$ which are coupled with a vertex in $U$. Clearly $U_{2} \cap \Gamma\left(U_{1}\right)=\emptyset$, because otherwise some coupling terminal is not connected to $\mathcal{T}$. Hence $\Gamma\left(U_{1}\right) \subseteq C_{2}$.

As our expander is $\frac{\beta+\gamma}{\beta-\gamma}$-good, it implies that either $\left|\Gamma\left(U_{1}\right)\right| \geq k+1-\left|U_{1}\right|$, or $\left|\Gamma\left(U_{1}\right)\right| \geq \frac{\beta+\gamma}{\beta-\gamma}\left|U_{1}\right|$.

We see that the first condition is not satisfied, as

$$
k-\left|U_{1}\right| \geq k-\left|U_{2}\right|=\left|C_{2}\right| \geq\left|\Gamma\left(U_{1}\right)\right| .
$$


Thus we can apply the second one to get

$$
\left|C_{2}\right| \geq\left|\Gamma\left(U_{1}\right)\right| \geq \frac{\beta+\gamma}{\beta-\gamma}\left|U_{1}\right| .
$$

Now we modify $\mathcal{T}$ to the new well-behaved ST $\mathcal{T}_{\text {new }}$ as follows: all vertices in $A_{1}$ and none in $A_{2}$ are in $\mathcal{T}_{\text {new }}$, and for any distinguished vertex $u$ which is labeled by literal distinct from $v$ and $\bar{v}$,

$$
u \in \mathcal{T}_{\text {new }} \Leftrightarrow u \in \mathcal{T} .
$$

We also connect the coupling terminals accordingly.

Applying formula (3) for well-behaved Steiner trees we obtain

$$
w(\mathcal{T})-w\left(\mathcal{T}_{\text {new }}\right)=\left(N-N_{\text {new }}\right) \beta+\left(M-M_{\text {new }}\right) \gamma .
$$

Clearly, $N-N_{\text {new }}=\left|C_{2}\right|-\left|U_{1}\right|$ and $M_{\text {new }} \leq M+\left|C_{2}\right|+\left|U_{1}\right|$, hence

$$
w(\mathcal{T})-w\left(\mathcal{T}_{\text {new }}\right) \geq\left(\left|C_{2}\right|-\left|U_{1}\right|\right) \beta-\left(\left|C_{2}\right|+\left|U_{1}\right|\right) \gamma=\left|C_{2}\right|(\beta-\gamma)-\left|U_{1}\right|(\beta+\gamma),
$$

which is nonnegative, by (5). Thus $w\left(\mathcal{T}_{\text {new }}\right) \leq w(\mathcal{T})$.

Now we apply the similar modification to $\mathcal{T}_{\text {new }}$ with another variable. It is easy to see that if we have done this for all variables, one after another, the result $\mathcal{T}^{*}$ is a standard tree for some assignment, with $w\left(\mathcal{T}^{*}\right) \leq w(\mathcal{T})$. Consequently, $w\left(\mathcal{T}^{*}\right)=\mathrm{OPT}$.

Theorem 2. Given an integer $d \geq 3$ and let $c(d)$ be a constant defined in (2). Let further an $(\alpha, \beta, \gamma)$-gadget with $\beta>\gamma>0$ and $\frac{\beta+\gamma}{\beta-\gamma}<c(d)$ be given. Then for any constant $r, 1<r<1+\frac{\gamma}{3 d+2 \alpha+3 \beta}$, it is NP-hard to approximate the Steiner Tree Problem within ratio $r$.

Moreover, if the gadget above is (uniformly) quasi-bipartite, the same inapproximability results apply to the (uniformly) quasi-bipartite instances of the STP as well.

Proof. Let an integer $d \geq 3$, an $(\alpha, \beta, \gamma)$-gadget and a number $r$ with the above properties be fixed.

We can choose and keep fixed from now on an $\varepsilon \in\left(0, \frac{1}{4}\right)$ such that

$$
r<1+\frac{(1-4 \varepsilon) \gamma}{3 d+2 \alpha+3 \beta+2 \varepsilon \gamma} .
$$

Let $k(\varepsilon)$ be an integer such that for any integer $k \geq k(\varepsilon)$ the conclusion of Theorem 1 holds. Since $\frac{\beta+\gamma}{\beta-\gamma}<c(d)$, we can consider and keep fixed from now on one $\frac{\beta+\gamma}{\beta-\gamma}$-good $d$-regular expander graph $H$ with $k$ by $k$ bipartition such that $k \geq k(\varepsilon)$. It will play a role of a constant in our (polynomial time preserving) reduction from NP-hard problem $P_{0}(\varepsilon, k)$ (respectively, $P_{1}(\varepsilon, k)$ ) to the problem of approximation STP within $r$. (Strictly speaking, we do not construct this reduction; we only show that there exists one. But this clearly suffices for proving 
NP-hardness.) Hence (with everything above fixed, including $k$ and $H$ ) we are ready to describe the reduction. Given an instance $L$ of the problem $P_{0}(\varepsilon, k)$ (respectively, $P_{1}(\varepsilon, k)$ ) with $n$ equations, whose optimum MAX of the maximal number of satisfiable equations is promised to be either at most $n\left(\frac{1}{2}+\varepsilon\right)$ or at least $n(1-\varepsilon)$, the reduction described above produces the corresponding instance of the STP problem. Since the assumptions of Lemma 1 are satisfied, the optimum OPT is achieved on a standard Steiner tree. Hence, using (3), the optimum OPT of the corresponding instance of the Steiner tree problem is

$$
\mathrm{OPT}=n \alpha+\frac{3}{2} n d+\frac{3}{2} n \beta+(n-\mathrm{MAX}) \gamma,
$$

which has to be now either at least $n \alpha+\frac{3}{2} n d+\frac{3}{2} n \beta+n\left(\frac{1}{2}-\varepsilon\right) \gamma$, or at most $n \alpha+\frac{3}{2} n d+\frac{3}{2} n \beta+n \varepsilon \gamma$.

Hence even the partial decision subproblem of the STP, namely the problem to distinguish between these two cases, is NP-hard. Consequently, since

$$
\frac{n \alpha+\frac{3}{2} n d+\frac{3}{2} n \beta+n\left(\frac{1}{2}-\varepsilon\right) \gamma}{n \alpha+\frac{3}{2} n d+\frac{3}{2} n \beta+n \varepsilon \gamma}=1+\frac{(1-4 \varepsilon) \gamma}{2 \alpha+3 d+3 \beta+2 \varepsilon \gamma}>r
$$

it is NP-hard to approximate the STP within $r$.

Moreover, it can be easily seen that if the gadget above is (uniformly) quasibipartite, our reduction produces (uniformly) quasi-bipartite instances of the STP, and the inapproximability results apply to those instances as well.

Theorem 3. Given an integer $d \geq 3$, denote $q(d)=\min \left\{\frac{c(d)-1}{2 c(d)}, \frac{1}{4}\right\}, r(d)=$ $1+\frac{q(d)}{3(d+1-q(d))}$, where $c(d)$ be a constant defined in (2). Then for any constant $r, 1<r<r(d)$, it is NP-hard to approximate the Steiner Tree Problem within ratio $r$.

In particular, since $c(6)>1.76222$ implies $r(6)>1.01063$, inapproximability within $1.01063\left(>\frac{96}{65}\right)$ follows for the STP.

Proof. Let an integer $d \geq 3$ and a number $r, 1<r<r(d)$, be fixed. We can find $\gamma \leq \frac{1}{4}$ with $\gamma<\frac{c(d)-1}{2 c(d)}\left(\right.$ i.e. $\left.\frac{1}{1-2 \gamma}<c(d)\right)$ and such that $r<1+\frac{\gamma}{3(d+1-\gamma)}$, and apply the Theorem 2 with $(0,1-\gamma, \gamma)$-gadget from Example 1 (with $\gamma$ as above and $\alpha=0$ and $\beta=1-\gamma)$.

Theorem 4. Given an integer $d \geq 3$ and denote by $r(d)=1+\frac{c(d)-1}{6 d \cdot c(d)+7 c(d)-1}$, where $c(d)$ be a constant defined in (2). Then it is NP-hard to approximate solution of (uniformly) quasi-bipartite STEINER TREe PROBLEM within ratio $r$, for any $r, 1<r<r(d)$.

In particular, since $c(7)>1.94606$ implies $r(7)>1.00791$, inapproximability within 1.00791 ( $\left.>\frac{128}{127}\right)$ follows for (uniformly) quasi-bipartite STP.

Proof. Let an integer $d \geq 3$ and a number $r, 1<r<r(d)$, be fixed. We can find $\gamma<\frac{c(d)-1}{2 c(d)}$ such that $r<1+\frac{\gamma}{3(d+3+\gamma)}$, and apply the Theorem 2 with the uniformly quasi-bipartite $(3+3 \gamma, 1-\gamma, \gamma)$-gadget from Example 2 (with $\gamma$ as above and hence $\alpha=3+3 \gamma$ and $\beta=1-\gamma)$. 
Remark. The methods of this paper provide a new motivation for the study of bounds for the parameters of expanders that provably exist. For our purposes we need not restrict ourselves to expanders that can be effectively constructed; the existence is enough. There is a substantial gap between the known upper and lower bounds for parameters of the best possible expanders. We believe that lower bounds on our expander constants $c(d)$ can be improved significantly. This would improve our inapproximability results. Another way to improve the results would be to provide the gadgets with better parameters.

\section{References}

1. Arora, S., Lund, C., Motwani, R., Sudan, M., Szegedy, M.: Proof verification an hardness of approximation problems. Proceedings of the 33rd Annual Symposium on Fundations of Computer Science, 1992, 14-23.

2. Bern, M., Plassmann, P.: The Steiner Problem with edge lengths 1 and 2. Information Processing Letters 32 (1989) 171-176

3. Chun, F. R. K.: Spectral Graph Theory. CBMS Regional Conference Series in Mathematics, American Mathematical Society, 1997, ISSN 0160-7642, ISBN 0-8218-0315-8.

4. Håstad, J.: Some optimal inapproximability results. Proceedings of the 28rd Annual Symposium on Theory of Computing, ACM, 1997.

5. Hougardy, S., Gröpl, C., Nierhoff, T., Prömel, H. J.: Approximation algorithms for the Steiner tree problem in graphs. In Steiner Trees in Industry, (X. Cheng and D.-Z. Du, eds.), Kluwer Academic Publishers, 2001, 235-279.

6. Karp, R. M.: Reducibility among combinatorial problems, In Complexity of Computer Computations, (Proc. Sympos. IBM Thomas J. Watson Res. Center, Yorktown Heights, N.Y., 1972), New York: Plenum 1972, 85-103.

7. Papadimitriou, C. H., Vempala, S.: On the Approximability of the Traveling Salesman Problem. Proceedings of the 32nd ACM Symposium on the theory of computing, Portland, 2000.

8. Robins, G., Zelikovsky, A.: Improved Steiner tree approximation in graphs. Proceedings of the Eleventh Annual ACM-SIAM Symposium on Discrete Algorithms $2000,770-779$.

9. Thimm, M.: On the Approximability of the Steiner Tree Problem. Proceedings of the 26th International Symposium, MFCS 2001 Mariánske Lázne, Czech Republic, August 27-31, 2001, Springer, Lecture Notes in Computer Science 2136 (2001) 678-689. 